\title{
Concurrent Inter-ONU Communications for Next Generation Mobile Fronthauls based on IMDD Hybrid SSB OFDM-DFMA PONs
}

Zhong, Zhuqiang; Jin, Wei; Jiang, Shan; He, Jiaxiang; Chang, Da; Hong, Yanhua; Giddings, Roger; Jin, Xianqing; O'Sullivan, M.; Durrant, T.; Trewern, J.; Mariani, G.; Tang, Jianming

\section{Journal of Lightwave Technology}

DOI:

10.1109/JLT.2021.3115573

Published: 01/12/2021

Peer reviewed version

Cyswllt i'r cyhoeddiad / Link to publication

Dyfyniad o'r fersiwn a gyhoeddwyd / Citation for published version (APA):

Zhong, Z., Jin, W., Jiang, S., He, J., Chang, D., Hong, Y., Giddings, R., Jin, X., O'Sullivan, M., Durrant, T., Trewern, J., Mariani, G., \& Tang, J. (2021). Concurrent Inter-ONU Communications for Next Generation Mobile Fronthauls based on IMDD Hybrid SSB OFDM-DFMA PONs. Journal of Lightwave Technology, 39(23), 7360-7369. https://doi.org/10.1109/JLT.2021.3115573

\footnotetext{
Hawliau Cyffredinol / General rights

Copyright and moral rights for the publications made accessible in the public portal are retained by the authors and/or other copyright owners and it is a condition of accessing publications that users recognise and abide by the legal requirements associated with these rights.

- Users may download and print one copy of any publication from the public portal for the purpose of private study or research.

- You may not further distribute the material or use it for any profit-making activity or commercial gain

- You may freely distribute the URL identifying the publication in the public portal ?
}

Take down policy

If you believe that this document breaches copyright please contact us providing details, and we will remove access to

the work immediately and investigate your claim. 


\title{
Concurrent Inter-ONU Communications for Next Generation Mobile Fronthauls based on IMDD Hybrid SSB OFDM-DFMA PONs
}

\author{
Z. Q. Zhong, W. Jin", S. Jiang, J. X. He, D. Chang, Y. H. Hong, R. P. Giddings, X. Q. Jin, M.
}

O'Sullivan, T. Durrant, J. Trewern, G. Mariani, and J. M. Tang Member, IEEE

\begin{abstract}
In PON-based mobile fronthauls, direct inter-ONU communications without passing end-user traffic to the OLT offer a promising solution for fulfilling thethe-stringent latency and bandwidth requirements of of $\mathbf{5 G}$ and beyond networks. In this paper, with slight modifications to at-the PON remote node, a concurrent inter-ONU and upstream communication technique is proposed and experimentally demonstrated in 101.6Gbit/s multipoint-to-point hybrid SSB OFDM - digital filter multiple access (DFMA) IMDD PONs over at a distance of $25 \mathrm{~km}$ SSMFs. Multiple gapless inter-ONU and upstream SSB signals ar aggregated by digital orthogonal filtering in each ONU transmitter. A single FFT operation is applied for demultiplex and demodulation in the OLT/ONU receivers. The results show that for both the inter-ONU and upstream transmissions, the optimum length of eptimal digital filters is -is-32, based on which the power penalties due to the fiber transmission and ONU channel interference are $<\mathbf{1 d B}$ and $<\mathbf{2 d B}$, respectively. For the inter-ONU communications, adaptive RF spectral assignments can effectively mitigate the Rayleigh and Brillouin backscattering effects and the upstream channel fading effect, thus giving rise to $>30 \%$ improvements in aggregated signal transmission capacity. In addition, detailed experimental investigations are alse undertaken of the trade-off between differential ONU optical launch power dynamic range and aggregated signal transmission capacity. An approximately 1dB increase in ONU launch powe dynamic range is achievable by reducing the aggregated signal transmission capacity by $\mathbf{5 G b i t} / \mathrm{s}$.
\end{abstract}

Index Terms-Inter-ONU communications, digital filter multiple access (DFMA), intensity modulation and direct detection (IMDD), passive optical networks (PONs).

\section{INTRODUCTION}

s a key network segment connecting centralized baseband A units (BBUs) and remote radio heads (RRHs) in radio

This work was supported in part by the DESTINI project funded by the ERDF under the SMARTExpertise scheme, and in part by the DSP Centre funded by the ERDF through the Welsh Government.

Z. Q. Zhong, W. Jin, S. Jiang, J. X. He, D. Chang, Y. H. Hong, R. P. Giddings, X. Q. Jin and J. M. Tang are with the School of Computer Science and Electronic Engineering, Bangor University, Bangor, LL57 1UT, UK. (email: z.zhong@bangor.ac.uk; w.jin@bangor.ac.uk; j.tang@bangor.ac.uk) and Z. Q. Zhong is also with the College of Science, Chongqing University of Technology, Chongqing 400054, China.

M. O'Sullivan is with Ciena Canada, Inc., 385 Terry Fox Drive, Ottawa, Ontario K2K 0L1, Canada.

T. Durrant and J. Trewern are with EFFECT Photonics LTD., Brixhan Laboratory, Freshwater Quarry, Brixham, Devon, England, TQ5 8BA, UK

G. Mariani is with EFFECT Photonics B.V., Kastanjelaan 400, 5617BC Eindhoven, Netherlands. access networks (RANs), mobile fronthauls (MFHs) play a crucial role in fulfilling thethe stringent high bandwidth, low latency, and ultra-dense connection requirements of $5 \mathrm{G}$ and beyond networks [1], [2]. However, current static MFHs mainly based on the common public radio interface (CPRI) are not spectrally and energetically efficient when transmitting digitized orthogonal frequency division multiplexing (OFDM) IQ waveforms at fixed bitrates [3]. Making matters even worse is the fact that the bandwidths required by current MFHs scale in proportion to the number of antenna ports [4], [5]. This considerably inhibits the RANs from utilizing highly desired advanced multi-antenna techniques such as massive multiple-input and multiple-output (MIMO) and beaming forming techniques. To effectively address these technical challenges, for baseband processing functionalities [4]-[6], the $3^{\text {rd }}$ Generation Partnership Project (3GPP) has released 8 functional split options, among which the low layer splits of Option 7-3, Option 7-2 and Option 6 attract industrial interests for implementingimplementing 5G and beyond MFHs [5]. To support flexible functional splits, the CPRI group has also released an enhanced CPRI (eCPRI) specification [7], based on which the resulting MFHs transmit digital signals (data is bit oriented or digitalized frequency OFDM IQ signals) via current IP or ethernet packet transport networks. However, such digital MFHs are still bandwidth inefficient.

To achieve bandwidth efficient MFHs, transmitting aggregated multiple mobile channels over analogue optical fronthaul links is highly desirable, and valuable work for multi-channel aggregation and de-aggregation has been reported [8]-[12], which can be grouped into Ttwo main categories are usually applied-including extra radio frequency (RF) component/electrical filter and digital signal processing (DSP). For the first category techniques, multi-channel aggregation and de-aggregation are realised by using extra RF components such as IQ up-converter or electrical filters [8]. On the other hand, for the second category techniques, use can be made of fast Fourier transform (FFT)/inverse FFT (IFFT)-based frequency division multiplexing (FDM) [9], code division multiplexing (CDM) [10], and digital domain up-conversion-based FDM [11], as well as digital shaping/matching filter (SF/MF) pairs-enabled digital filter multiplexing (DFM) [12]. In comparison with the first category techniques, the DSP-enabled second category techniques are more attractive due to their cost effectiveness and inherent 


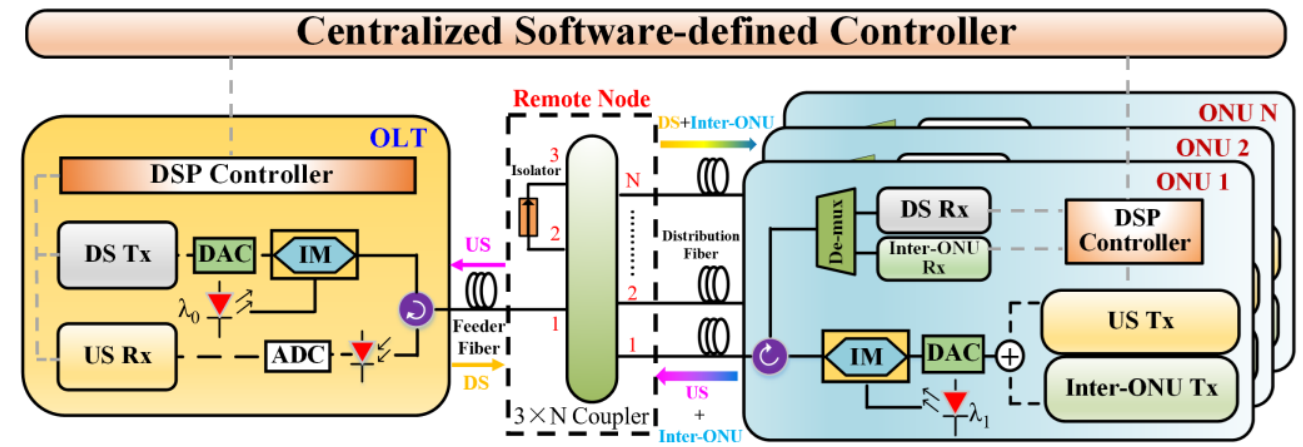

Fig. 1 Schematic diagram of the proposed IMDD hybrid SSB OFDM-DFMA PONs supporting concurrent Inter-ONU communications. DAC/ADC: digital-to-analogue/analogue-to-digital conversion, IM: intensity modulation, US: upstream, DS: downstream, Tx: transmitter, Rx: receiver. De-mux: digital-to-analogue/analogue-10
wavelength de-multiplexer.

digital-domain operationat reconfigurability and flexibility [12]. However, to practically implement the DSP-based second category techniques, frequency guard band [13], frequency domain windowing techniques [14], or channel interference cancellation techniques [15] may be required to significantly reduce the channel interferences. Furthermore, additional DSP may also be essential for further improving achieving flexible numerology, excellent channel bandwidth elasticity, channel count-independent performance, excellent adaptability to transmission system impairments and transparency to flexible RAN low layer splits. Given the fact that intensity modulation and direct detection (IMDD) passive optical networks (PONs) are promising for cost-effectively providing broadband connectivity for MFHs [16], [17], to solve the aforementioned technical challenges, hybrid single sideband (SSB) OFDM-digital filter multiple access (DFMA) PONs based on IMDD [18] have been reported, where Ffor upstream communications, multiple gapless SSB OFDM signals produced without performing the Hilbert transform operations are passively combined in the remote node, and simultaneously separated and demodulated by a single FFT operation in the OLT without performing the matching filtering operation.

On the other hand, 5G and beyond networks are expected to support a large diversity of user-oriented services such as virtual private broadband connections, multimedia resource sharing and collaborative computing [19], [20]. Such highly interactive services/applications are bandwidth hungry an latency sensitive. The maximum transport network latency associated with the MFHs is primarily determined by the RAN internal processes time constraints such as HARQ loop, scheduling, etc [21]. The low end-to-end latency required by $5 \mathrm{G}$ determines the maximum allowable MFH latency to be in the range of $250 \mu \mathrm{s}$ [5]. For a typical $20 \mathrm{~km}$ fronthaul with $\sim 5 \mu \mathrm{s} / \mathrm{km}$ fiber propagation delay, the roundtrip time for the fiber propagation is $\sim 200 \mu$ s. Obviously, the fiber propagation-induced time delay plays a dominant role in determining the overall end-to-end latency. Considering the case where a large amount of data is exchanged between different end-users in a single MFH network infrastructure, to provide interactive services in conventional PON-based MFHs, end-user traffic has to be transmitted to the OLT via an upstream link, then re-modulated onto a downstream carrier and finally routed to the destined ONUs at the radio site. This unavoidably results in unnecessary bandwidth consumption, additional transmission latency and extra signal processing in the OLT. Thus, it is highly advantageous if end-to-end latency reductions can be realized by providing end-users with direct communications without passing traffic to the OLT through the long feeder fiber. However, previously reported research work, capable of achieving direct communications between ONUs, requires need the introduction-of extra optical filters [22] or multiple additional transceivers [23], leading to this means huge modifications to conventional PON architectures-are necessary. In addition, theisse works also has ve limited flexibility and transparency to future converged networks, where a wide diversity of key network operation features such as various multiple access techniques are envisaged to be accommodated simultaneously [22]-[24]. More importantly, these techniques suffer from a significantly reduced upstream bandwidth utilization efficiency as the upstream and inter-ONU communications channels share the same spectral regions [25].

To address the aforementioned challenges in a cost-effective manner, very recently, by making simple alterations to the remote node of our previously proposed hybrid single sideband (SSB) OFDM DFMA PONs [18], concurrent inter-ONU and upstream transmissions have been experimentally demonstrated [26], for the first time, in 25km IMDD-based hybrid SSB OFDM-DFMA PONs with an aggregated net signal transmission capacity of $101.6 \mathrm{Gbit} / \mathrm{s}$. Compared with the previously reported direct ONU communication techniques, the proposed PONs has the following advantages: 1) slight minor modifications to at the remote nodes, this which maintains the PON's passive characteristics, 2) DSP-enabled adaptive RF spectral assignments and DSP-based dynamic variations in both inter-ONU communication channel count and/or upstream channel count without requiring either remote node reconfigurations or and-extra transceiver electrical/optical components, 3 ) inherent compatibility to flexible RAN's low 
layer splits, 4) digital filtering-enhanced numerology flexibility, and 5) excellent compatibility with existing high layer low latency schemes.

In this paper, further experimental explorations of the proposed inter-ONU communication techniques are have been undertaken in $25 \mathrm{~km}$ IMDD hybrid OFDM-DFMA PONs. As a significant extension of our previously published work [26], in this paper special attention is given to the following two aspects: 1) investigations of Rayleigh and Brillouin backscattering effects and 2) the use of using DSP-enabled adaptive RF spectral assignments to effectively reduce the impacts of the effects of channel fading and backscattering effects on aggregated transmission capacity. Our experimental results show that the proposed hybrid SSB OFDM DFMA PONs supporting concurrent inter-ONU communications have the following excellent features:

1) Excellent robustness against fiber transmission impairments. The fiber transmission-induced power penalties for upstream and inter-ONU communications are $<1 \mathrm{~dB}$ and $<0.2 \mathrm{~dB}$ respectively.

2) Digital filtering-achieved significant channel interference reductions. The ONU channel interference-induced powe penalties are $<2 \mathrm{~dB}$ for both upstream and inter-ONU communications.

3) Simple digital filter reconfigurations. Both upstream and inter-ONU communications require similar optimum digita filter designs, which are independent of channel frequency location.

4) Considerable spectral efficiency enhancements because DSP-enabled adaptive RF spectral assignments reduces the channel fading and backscattering effects. In comparison with the case of assigning all available RF bandwidths for upstream (inter-ONU) communications, the DSP-enabled adaptive RF spectral assignments improve the aggregated transmission capacity by $\sim 31.4 \%(\sim 1.34 \%)$.

In addition, the trade-offs between ONU launch power dynamic range and aggregated transmission capacity are also is experimentally investigated. The results show that by slightly reducing the aggregated transmission capacity, a $>5 \mathrm{~dB}$ increase in ONU launch power dynamic range is achievable.

\section{PRINCIPLE OF THE IMDD HYBRID SSB OFDM-DFMA} PONS WITH CONCURRENT INTER-ONU COMMUNICATIONS

The schematic diagram of the proposed IMDD hybrid SSB OFDM-DFMA PON supporting concurrent inter-ONU communications is shown in Fig. 1. In comparison with our previously reported IMDD hybrid OFDM-DFMA PONs incorporating an 1:N passive optical coupler-based remote node[18], a slight modification is made in this PON architecture, i.e., a 3:N passive optical coupler and an optical isolator are used in the remote node. For upstream and inter-ONU communications, the SSB OFDM signal bands for upstream and inter-ONU communications are combined in each ONU in the digital domain. In Fig.2, the detailed OFDM signal generation process and digital filtering process are illustrated, which are discussed in the next paragraph. After passing through a digital-to-analogue converter (DAC), in each $\mathrm{ONU}$, the generated electrical signal containing both upstream
SSB signals and inter-ONU communication SSB signals is sent to an intensity modulator (IM) to perform electrical-to-optical conversion and then transmitted to the remote node through the corresponding distribution fiber. As the remote node includes a $3 \times \mathrm{N}$ optical coupler and an optical isolator, the combined optical signals from different ONUs are transmitted to the OLT via the feeder fiber. The remaining part of the combined optical signals are redirected and broadcasted to each ONU via the remote node to achieve inter-ONU communications. At the OLT, the optical signal is fed to a PIN detector to perform square-law photo detection, then digitized by an analogue-to-digital converter (ADC), and finally demultiplexed and demodulated utilizing a DSP procedure shown in Fig. 3. At the ONUs, the downstream and inter-ONU signals are broadcasted to all the ONUs. To effectively avoid the interferences between the inter-ONU signals-and downstream signals-interferences, similar to existing PONs, different wavelengths may be deployed for the inter-ONU and downstream communications, -as shown in Fig. 1, the be deployed with different wavelengths, separated by wavelength de-multiplexers and then demodulated via their receivers, respectively. The inter-ONU signal demodulation DSP procedures are similar to the upstream signal demodulation DSP procedures, which are depicted in Fig. 3. It should be noted that in the proposed PONs, each ONU's upstream and inter-ONU communication OFDM SSB signals locate at different RF regions, but they are modulated on the same wavelength. Theis inter-ONU communication technique is can also be applicable in the hybrid doubled sideband (DSB) OFDM-DFMA PONs [18] where transmitting -real-valued OFDM signals or discrete multi-tone signals are transmitted over multiple orthogonal channels. Compared with the DSBformer case, techniques, the utilization of the SSB signals can further improve the the hybrid SSB OFDM-DFMA PONs have higher -spectral efficiency and improved-robustness against transmission system impairments. In this paper, we will focus on the more challenging upstream and inter-ONU communications of the proposed PONs only.

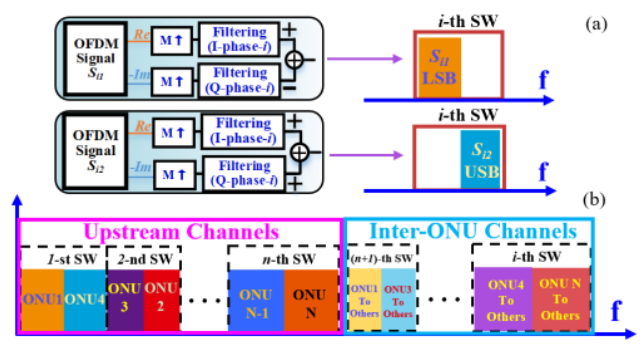

Fig. 2 Digital filtering processing in ONUs and corresponding signal spectral locations. $\mathrm{M} \uparrow$ : digital-domain up-sampling operation by a factor of $\mathrm{M}, \mathrm{SW}$ : sub-wavelength

Fig. 2 shows the digital filtering procedure processing for generating the SSB OFDM signals at desired RF regions and the channel spectral allocations. Here, the 'SSB OFDM' refers to as the electrical SSB OFDM signals, and their corresponding 
optical signals produced after - After-intensity modulation are referred to as, the produced optical signal is "DSB in optical domain". As seen in Fig. 2(b), for achieving a high spectral efficiency, the whole available RF spectral region is split into two spectral sections, and the low (high) spectral regionsection is allocated for upstream (inter-ONU) communications with long (short) latency. Each spectral region section-contains multiple gapless sub-wavelengths (SWs) and each SW can deliver two independent gapless SSB OFDM signals. In a SW, the low and high frequency SSB signals respectively correspond, respectively, to the lower sideband and upper sideband (LSB and USB) conveyed by the SW. Fig. 2(a) presents the detailed digital filtering process for generating the LSB (conveying complex OFDM signal $S_{i l}$ ) and USB (conveying complex OFDM signal $S_{i 2}$ ), where the subscript $i$ represents the $i$-th SW. In generating the complex $S_{i 1}$ and $S_{i 2}$ OFDM signals, a SSB OFDM signal generation process [18] is adoptedused, where half of the OFDM subcarriers are set at zeros. As a direct result of the above channel DSPs, the real part and the (-1)-multiplied imaginary part of each produced complex OFDM signal form a Hilbert transform pair [27]. Based on the digital filtering process in Fig. 2(a), the LSB and USB of the $i$-th SW can be described as,

$$
\begin{aligned}
& S_{S S B-i q}(t)=M \uparrow\left\{\operatorname{Re}\left(S_{i q}(t)\right)\right\} \otimes h_{i}^{I}(t)+ \\
& (-1)^{q} M \uparrow\left\{-\operatorname{Im}\left(S_{i q}(t)\right)\right\} \otimes h_{i}^{Q}(t)
\end{aligned}
$$

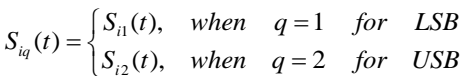

where $S_{i I}(t)$ and $S_{i 2}(t)$ are two independent SSB OFDM signals, $\mathrm{M} \uparrow$ represents the $\mathrm{M} \times$ up-sampling operation and the up-sampling factor $M$ is the parameter can be used to adjust achieve different channel counts and ehange the bandwidths for fixed DAC/ADC sampling speeds-of a specific channel. As a direct result, As such, adaptively and flexible-selecting an optimum up-sampling factor-selections - for a given each channel is effective in the main andy varying achieving flexible channel count variations and delivering bandwidth elasticity. $\left\{h_{i}^{I}(t), h_{i}^{Q}(t)\right\}$ are the $i$-th pair of the orthogonal digital shaping filters and their superscript $I$ and $Q$ stand for the in-phase/quadrature-phase digital filter type $\otimes$ represents the circular convolution operation.

The upstream and inter-ONU communications have simila SSB signal demodulation procedures, as illustrated in Fig. 3 After direct detection and an AC-coupled ADC, serial-to-parallel (S/P) conversion and cyclic prefix (CP) deletion are performed. Then a $N$-point $(N=B \times M)$ FFT operation is applied to demultiplex and demodulate the received multiple SSB OFDM signals, here $B$ is the IFFT size used for generating generation of the OFDM signals. In the sideband identification operation, the $N / 2$ subcarriers in the positive frequency bin are grouped into $M / 2$ sub-groups each corresponding to a SW. As such, the $B / 2$ low frequency subcarriers of each sub-group correspond to the LSB of the corresponding SW, and the remaining subcarriers of the same sub-group correspond to its USB counterpart. The OLT and ONUs receivers can use the above sideband identification process to identify respectively their desired channels for further signal demodulation. In the sideband data processing, a conjugation and a subcarrier reverse ordering operation are successively performed on the subcarriers contained in the LSB signals, while no extra DSP operations are needed for the USB signals. Finally, the conventional OFDM signal equalization and decoding processes can be applied for all the OFDM subcarriers without requiring extra signal recovery DSP algorithms.

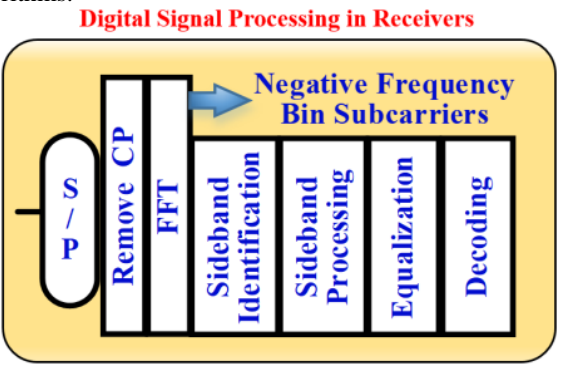

Fig. 3 Matching filter-free SSB signal demultiplexing and recovery process in upstream and inter-ONU communication receiver DSPs. S/P: serial-to-parallel conversion, CP: cyclic prefix, FFT: fast Fourier transform.

From the above analysis, it is easy to understand that the central frequency of the orthogonal digital shaping filter determines the corresponding digitally filtered or resulting SSB signal's frequency locations. As a result, by adaptive orthogonal digital filter allocations under the centralized software-defined networking (SDN) controller, each ONU can generate its upstream SSB signals and inter-ONU SSB signals at the desired RF frequency regions without causing contention with other ONUs. In addition, an up-sampling factor of $M$ leads to $M / 2$ orthogonal shaping filter pairs, therefore arbitrary channel counts are achievable by utilizing different up-sampling factors. By adaptively assigning the digital filters for upstream and inter-ONU communications, these channels can be flexibly allocated to upstream and inter-ONU communications thus enabling the dynamic sharing of available spectral regions. the channels. Due to the digital filtering process, each SW is allowed to flexibly use different OFDM numerologies or parameters including $\mathrm{CP}$ and subcarrier frequency spacing. For different numerologies, from the above analysis shows that -multiple FFT operations each corresponding to a numerology have to be performed in the receiver DSP procedures, and then the subcarrier identification processes can be conducted for the channels with the identical numerology after the corresponding FFT operations. The remaining receiver DSP procedures including sideband processing and equalization are similar to those mentioned above.

III. EXPERIMENTAL DEMONSTRATIONS OF CONCURRENT INTER-ONU AND UPSTREAM COMMUNICATIONS IN IMDD HYBRID SSB OFDM-DFMA PONS

The experimental setup is constructed using off-the-shelf 
optical/electrical devices and the impacts of practical fiber transmission impairments, digital filter characteristic variations as well as the ONU channel interference effect on the bit error ratio (BER) performances are thoroughly explored experimentally. In addition, the Rayleigh and Brillouin backscattering effects are also measured, based on which the capability of the DSP-enabled adaptive RF spectral assignments, for reducing both the channel fading effects and

$\begin{array}{lc}\text { Up-sampling factor } & 4 \\ \text { Modulation formats } & \text { QPSK to 64QAM } \\ \text { Cyclic prefix ratio } & 1 / 16 \\ \text { Clipping ratio } & 11 \mathrm{~dB} \\ \text { Digital filter length } & 32 \\ \text { DAC/ADC sampling speed } & 60 / 64 \mathrm{GS} / \mathrm{s} \\ \text { Overall signal bandwidth } & 24 \mathrm{GHz} \\ \text { ONU1 wavelength } & 1549.32 \mathrm{~nm} \\ \text { ONU2 wavelength } & 1550.12 \mathrm{~nm} \\ \text { Thorlabs transmitter MZM } \mathrm{V}_{\pi} & \sim 3.1 \mathrm{~V}\end{array}$

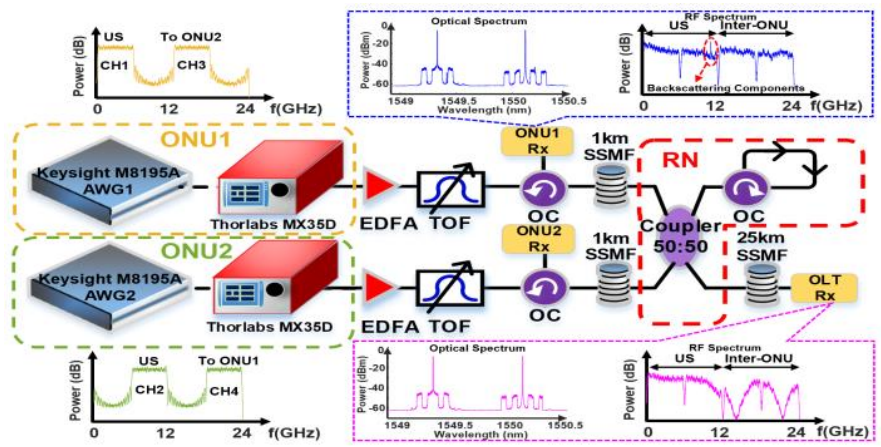

Fig. 4 Experimental setup of concurrent inter-ONU and upstream communications in IMDD hybrid SSB OFDM-DFMA PONs. CH1/2/3/4: channel 1/2/3/4, US: upstream, EDFA: Erbium-doped fiber amplifier, TOF: tunable optical filters, OC: optical circulator, SSMF: standard single-mode fiber, RN: remote node, Rx: receiver

\section{the backscattering effects, are verified.}

\section{A. Experimental Setup and Parameters}

The experimental setup of concurrent inter-ONU and upstream communications in the IMDD hybrid SSB OFDM-DFMA PONs is illustrated in Fig. 4, where two ONUs are adopted with an external clock and a trigger signal used-for ONU timing synchronization. In each ONU, an arbitrary waveform generator (AWG, Keysight M8195A) operating at 60GS/s@8-bit is utilized for generating transmitted analogue signals. In each ONU DSP, two independent complex OFDM signals are first produced utilizing the DSP procedure signal processing already stated in Section II. To explore the maximum transmission capacity, adaptive bit-loading and power-loading are utilized for each OFDM signal with subcarrier modulation formats varying from QPSK to 64-QAM. The resulting subcarrier bit allocation -loading profiles scheme corresponding to for achieving the the 101.6Gbit/s signal transmissions reported here -is presented in Fig. 5. After generating the complex OFDM signals, as described in Fig. 2, the real and imaginary parts of each OFDM signal are digitally up-sampled by a factor of $M=4$ and digitally filtered by orthogonal digital shaping filters, which are square-roo raised-cosine filters constructed utilizing Hilbert-pair approaches with a digital filter length of 32 and an excess of bandwidth factor of 0 . The other key experimental parameters are listed in Table 1.

\begin{tabular}{lc}
\multicolumn{2}{c}{ Table 1. Key Experimental Parameters } \\
\hline \multicolumn{1}{c}{ Parameter } & Value \\
\hline IFFT/FFT size & $32 / 128$ \\
Data-bearing subcarriers & 15
\end{tabular}

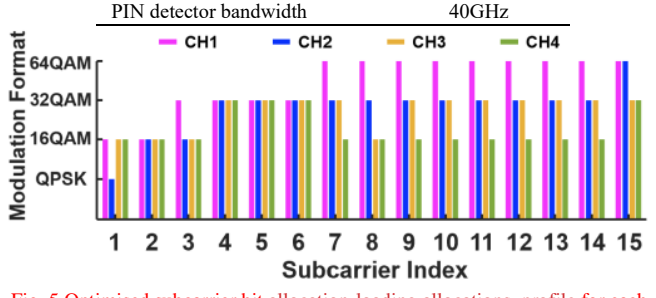

Fig. 5 Optimised subcarrier bit allocation-loading allocations- profile for each channel.

After the digital filtering process, a digital-domain time delay operation is applied for each OFDM band to adjust the channel timing offset. Finally, in each ONU, after digitally combining two digitally filtered OFDM signals, a $1.25 \times$ oversampling operation and a signal clipping operation are performed successively. As a result, the overall signal bandwidth is $24 \mathrm{GHz}$ and shared by a total of 4 OFDM channels with each OFDM channel having a $6 \mathrm{GHz}$ bandwidth. The higher frequency $(>12 \mathrm{GHz})$ channels suffer the severe channel fading effect after transmitting through the feeder fiber transmissions, as shown in the OLT RF spectrum of Fig. 4, thus $\mathrm{CH} 1$ and $\mathrm{CH} 2$ are assigned to convey upstream traffic, while $\mathrm{CH} 3$ and $\mathrm{CH} 4$ are assigned for the relatively short distance inter-ONU communications. As a result, a high overall spectral efficiency is achievable by adaptively allocating the channels for both upstream and inter-ONU communications. Following the AWGs, two transmitters (Thorlabs MX35D) each incorporating with-a $15 \mathrm{kHz}$ linewidth laser sources and- a 
$35 \mathrm{GHz}$ lithium niobate $\left(\mathrm{LiNbO}_{3}\right)$ Mach-Zehnder modulators inside- are employed to perform realize-electrical-to-optical conversion. The ONU central wavelength spacing is set to be $\sim 100 \mathrm{GHz}$ to minimize the optical beating interference (OBI) effect [28]. After passing through the EDFAs, $0.8 \mathrm{~nm}$ bandwidth tunable optical filters (TOF), optical circulators and $1 \mathrm{~km}$ standard single mode fibers (SSMFs), the two ONUs' optical signals with similar optical powers of $\sim 6 \mathrm{dBm}$ are passively coupled in the remote node. For simplicity, the remote node is constructed by a 50:50 coupler and an optical circulator. The combined optical signal is transmitted to the OLT through the lower output port of the coupler with a fixed optical power of $\sim 6 \mathrm{dBm}$. On the other hand, the combined optical signal can also be redirected and delivered to each ONU at the upper output port of the optical coupler. At the receivers, a 40GHz PIN photodetector (Thorlabs RXM40AF) performs the optical-to-electrical conversion, which is followed by and a $30 \mathrm{GHz}$ low-pass radio frequency (RF) filter is used-to remove the out-of-band noise. The received electrical signals are digitalized by a digital sampling oscilloscope (Keysight UXR0402A) with a sampling speed of $64 \mathrm{GS} / \mathrm{s}$ and then delivered to a PC to perform off-line signal demodulation. As discussed in Section II, the ONU and OLT receivers employ similar signal demodulation DSP procedures. In the this experiments, the signal demodulation procedures include signal re-sampling [29], frame synchronization, serial-to-parallel (S/P) conversion, cyclic prefix deletion, 128-point FFT operation, sideband identification, sideband data processing, conventional OFDM subcarrier equalization and data decoding. In the sideband identification process, the subcarriers in each channel are identified and the ONUs and OLT can easily select their desired channels for signal demodulation. i.e. the OLT selects the upstream channels of $\mathrm{CH} 1$ and $\mathrm{CH} 2$ and $\mathrm{ONU} 1$ (ONU2) only demodulates $\mathrm{CH} 4 \quad(\mathrm{CH} 3)$ for the inter-ONU communications. In the sideband data processing, due to the conjugate symmetry property of the sidebands, a conjugation operation and a subcarrier reverse ordering operation must be performed only for both- $\mathrm{CH} 1$ and $\mathrm{CH} 3$-nly.
B. Upstream and Inter-ONU Transmission Performances
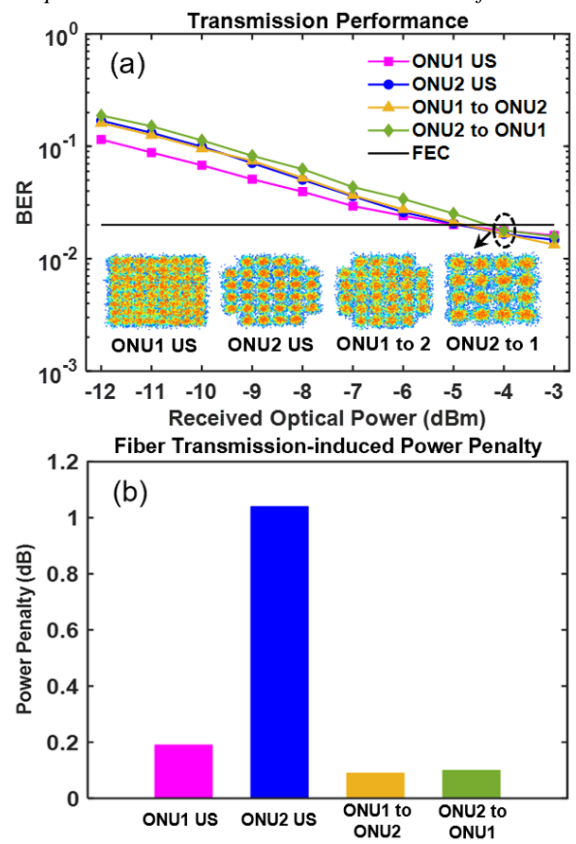

Fig. 6 Measured BER transmission performances (a) and fiber transmission-induced power penalty (b)

Utilizing the experimental setup and parameters presented in Section III.A, the measured upstream and inter-ONU transmission BER performances as well as the fiber transmission induced power penaltiesy are plotted in Fig. 6. In this paper, a $20 \%$ overhead soft-decision forward error correction (SD-FEC) threshold at a BER of $2 \times 10^{-2}$ is considered [30] and the fiber transmission-induced power penalty is defined as the minimum received optical power differences for achieving a-BER $\underline{s}$ at the FEC limit before and after fiber transmission. Due to the adaptive bit-loading and power-loading, all the channels have similar BER performances. Fig. 6(a) shows the 64-/32-/16-QAM constellations of the corresponding channels when the received optical powers (ROP) are isfixed at $-4 \mathrm{dBm}$. In addition, the fiber transmission-induced power penalties for $\mathrm{CH} 1$ and inter-ONU communication channels are $<0.2 \mathrm{~dB}$, while for $\mathrm{CH} 2$, the fiber transmission-induced power penalty increases to $1 \mathrm{~dB}$. The larger power penalty suffered by $\mathrm{CH} 2$ is mainly due to the strong higher channel fading effect over the $25 \mathrm{~km}$ feeder fiber, which this can be clearly observed from the RF spectrum in Fig. 4. Nevertheless, the impact of practical transmission system impairment/nonlinearity on receiver sensitivity degradations are almost negligible. 
C. Impacts of Digital Filter Characteristic Variations

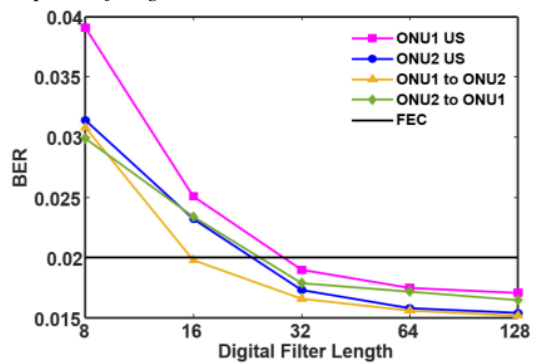

Fig. 7 Impact of ONU-embedded digital shaping filter lengths on BER performances for a received optical power of $-4 \mathrm{dBm}$.

In the hybrid OFDM-DFMA PONs, the optimum digital filter lengths must be determined to achieve sufficiently low BER performance but without incurring excessive DSP complexity. Generally speaking, a long digital filter length leads to improved transmission performances, however it inevitably causes an undesirable increase in digital filter DSP complexity. Thus, there is a trade-off between digital filter DSP complexity and the corresponding transmission performances. To identify the optimum digital filter length for achieving the targeted performances for a given practical application scenario, the impact of digital filter characteristic variations on BER performances is explored based on the an experimental setup similar to that used in obtaining Fig. 6. For digital filter lengths varying from 8 to 128 , the measured BER performances are plotted in Fig. 7. It can be observed that for all the transmission channels, when the-digital filter lengths are is-less than 32 , the BERs of for most of the channels, their BERs are larger than the FEC limit, because of short digital filter length-induced increase in digital filter frequency response ripples and power leakages [28]. While the BERs_performances_become almost flat when the digital filter length varies from 64 to 128 Therefore, by considering the transmission performances and as well as the digital filter DSP complexity for in-each ONU, the optimum digital filter length of is 32 is chosen for both upstream and inter-ONU communications, which is utilized throughout the paper.

D. Impacts of ONU Channel Interference Effect

For multi-channel communications, the ONU channel interferences play a key role in determining the practically achievable transmission performances. To explore the channe interferences between the two involved ONUs in the experimental setup adopted in Section III.A, the upstream and inter-ONU BER performances of one ONU with the other ONU electrical signals being switched on/off are plotted as a function of received optical power in Fig. 8. For upstream transmissions, as shown in the Fig. 8(a), when switching off the channels of particular ONU, the BER curve of the other ONU's upstream channel is left-shifted by $\sim 2 \mathrm{~dB}$. As such, for upstream channels, ONU channel interference-induced power penalties are $\sim 2 \mathrm{~dB}$. Meanwhile, the ONU channel interference-induced power penalties for inter-ONU communication are $\sim 1.7 \mathrm{~dB}$. Such power penalties may be further reduced by finely optimizing the subcarrier bit-loading profile-loading [18]
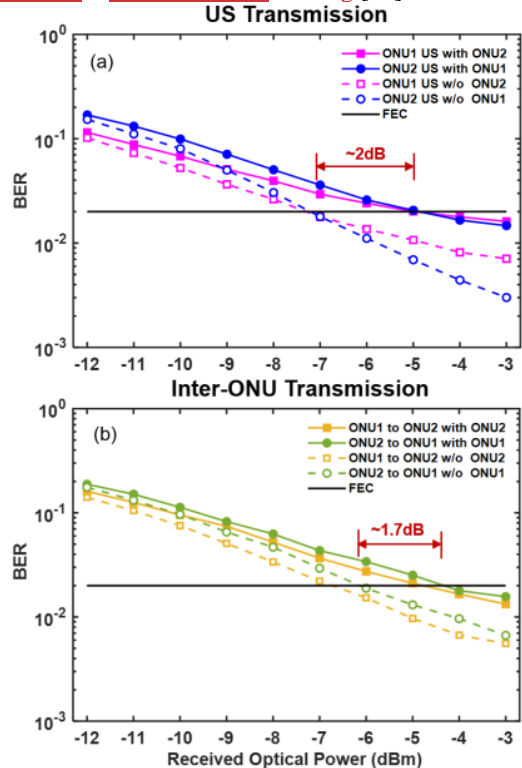

Fig. 8 Impact of ONU channel interference on US (a) and inter-ONU transmission performances $(\mathrm{b})$.

E. Measurements of the Rayleigh and Brillouin Backscattering Effect

Due to the utilization of the same optical wavelength for transmitting both upstream and inter-ONU traffic over the same $\mathrm{PON}$ infrastructure, the inter-ONU signals suffer from various backscattering effects mainly including the Rayleigh backscattering effect and the Brillouin backscattering effect. Therefore, it is essential to investigate these backscattering effects in the proposed IMDD hybrid SSB OFDM-DFMA PONs to improve the inter-ONU communication transmission performances.

In order to quantitively analyse the above two backscattering effects, a new experimental setup, as shown in Fig. 9(a), is utilised with the same devices adopted in Fig. 4. For simplicity, only ONU1 is considered where CH1 and CH3 OFDM signals, with their spectral locations shown in Fig. 4, are modulated onto a wavelength at $1549.32 \mathrm{~nm}$. The test fibers are SSMFs with their fiber lengths of $1 \mathrm{~km}$ and $25 \mathrm{~km}$, which representeorresponds to the distribution fiber and feeder fiber considered in the experimental setup in Fig. 4, respectively. An ultra-high resolution optical spectrum analyzer (OSA, Apex AP2070A) is used to record the details of the backscattered ing lights. Fig. 9(b) illustrates the optical spectra of Rayleigh and Brillouin backscattering components when a fiber launch power is $6 \mathrm{dBm}$. We can find that the overall backscattered optical signal powers containing both the Rayleigh and Brillouin backscattering components for the $25 \mathrm{~km}$ SSMF is $\sim 15 \mathrm{~dB}$ higher than that from the $1 \mathrm{~km}$ SSMF. For the considered 
fiber launch power of $6 \mathrm{dBm}$, the $25 \mathrm{~km}(1 \mathrm{~km}) \mathrm{SSMF}$ introduced Rayleigh backscattering component has an optical power of $-27 \mathrm{dBm}(-38 \mathrm{dBm})$. For the Brillouin backscattering effect, there exist two peaks corresponding to the Stokes and anti-Stokes components due to the inelastic interaction between the electromagnetic waves and crystalline lattice waves. The frequency shifts between the optical wavelength launched into the fiber and its Brillouin backscattering components are about $10.8 \mathrm{GHz}$ for the considered SSMFs.

Next, the backscattering power versus fiber launch power for the considered $1 \mathrm{~km}$ and $25 \mathrm{~km}$ SSFM are shown in Fig. 9(c). When the fiber optical launch power is $\angle 6 \mathrm{dBm}$, the powers of the Rayleigh and Brillouin backscattering components increase nearly linearly with the increasing e of fiber launch powers. This is because for small fiber launch powers, the majority of Brillouin backscattering is from the spontaneous Brillouin scattering process. However, for the $25 \mathrm{~km}$ SSFM, when the fiber launch powers are above $6 \mathrm{dBm}$, the stimulated Brillouin scattering becomes dominant, and a large amount of its backscattering energy contribute to the Brillouin Stokes component, which causes a rapid increase in the Brillouin Stokes component. When the fiber launch powers are $>8 \mathrm{dBm}$, the Brillouin Stokes component power becomes larger than the frequency region from $0 \mathrm{GHz}$ to $12 \mathrm{GHz}$ is allocated for the upstream communications, while the high RF frequency region from $12 \mathrm{GHz}$ to $24 \mathrm{GHz}$ is allocated to the inter-ONU communications.

IV. DifFERENTIAL ONU Optical LAUNCh POWER DyNAMIC RANGE

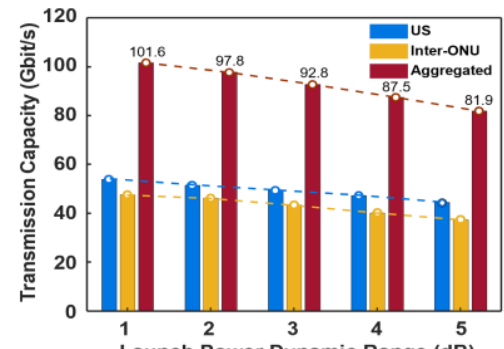

Launch Power Dynamic Range (dB)

Fig. 10 Transmission capacity versus differential ONU optical launch power dynamic range when received optical powers are fixed at $-3 \mathrm{dBm}$.

It is well known that various aggregated signal transmission
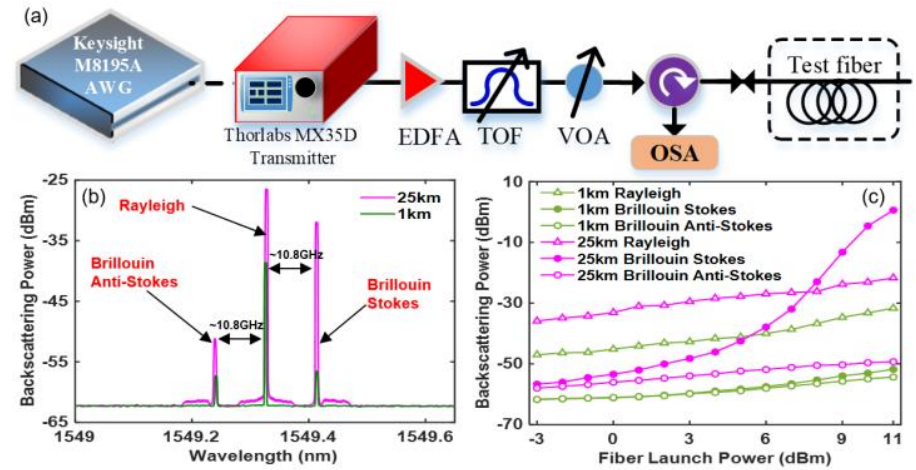

Fig. 9 (a) Experimental setup of Rayleigh and Brillouin backscattering measurements, (b) optical spectra of Rayleigh and Brillouin backscattering when fiber launch power is $6 \mathrm{dBm}$ and (c) backscattering power versus fiber launch power for $1 \mathrm{~km}$ and $25 \mathrm{~km}$ SMF. OSA: optical spectrum analyzer.

Rayleigh backscattering power.

Based on the above discussions, for lowering the backscattering effects in the proposed PONs, the feeder fiber launch power should not exceed $6 \mathrm{dBm}$, which can also reduce the fiber transmission nonlinearity effect. $\%$ As shown in ONU1's RF spectrum of Fig. 4, an undesired peak occurs at $\sim 10.8 \mathrm{GHz}$ arising from the beating of the backscattering components and inter-ONU optical signals. Such an electrica peak can destroy the OFDM subcarriers in the its-vicinity of fo the inter-ONU communications spectral region, but it does not occur in the upstream communications spectral region. Therefore, to mitigate both the backscattering effects on the inter-ONU communications and the channel fading effects on the upstream communications, the RF spectral regions for both the upstream and inter-ONU communications need to be appropriately selected suitably assigned for practical applications. To achieve this goal, in this paper, the low RF capacities can result in different differential ONU optical launch power dynamic ranges [18]. For the proposed IMDD hybrid SSB OFDM-DFMA PONs with concurrent inter-ONU communications, the differential ONU optical launch power dynamic ranges versus various signal transmission capacities are measured and the results are shown in Fig. 10, where adaptive bit-loading is used in each OFDM signal. It can be seen that, for an aggregated signal transmission capacity of 101.6Gbit/s, the differential ONU launch power dynamic range is only $\sim 1 \mathrm{~dB}$ and every $1 \mathrm{~dB}$ increase of the differential ONU launch power dynamic range gives rise to $\sim 5 \mathrm{Gbit} / \mathrm{s}$ reductions in aggregated transmission capacity. This reveals that for the proposed IMDD hybrid SSB OFDM-DFMA PONs supporting concurrent inter-ONU communications, arbitrary differential ONU launch power dynamic ranges can be made available by slightly compromising the aggregated upstream signal 
transmission capacity. It is also interesting to point out that the upstream signal transmission capacity is a little higher than that of the inter-ONU communications. The reason behind this is that, due to the transmission system impairments/nonlinearity, the inter-ONU signals at higher RF frequencies suffer lower signal-to-noise ratios (SNR) in comparison with the high frequency upstream signals.

\section{SPECTRAL EFFICIENCY ENHANCEMENT VIA DSP-ENABLED} ADAPTIVE RF SPECTRAL ASSIGNMENTS
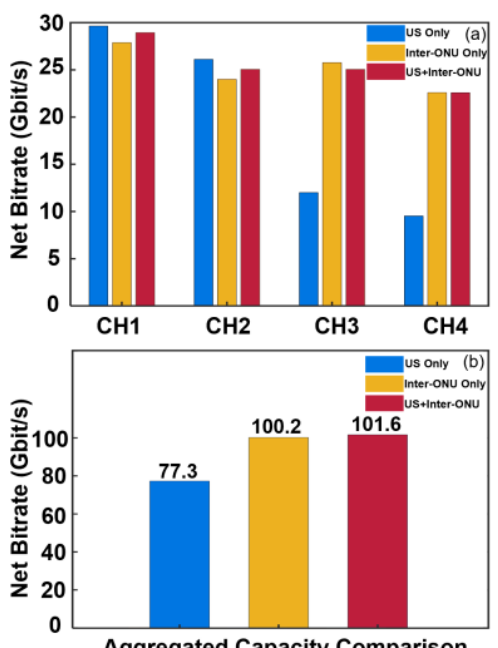

Aggregated Capacity Comparison

Fig. 11 (a) Transmission capacity of each channel and (b) their resulting aggregated transmission capacities in three cases.

As stated in Section III.E, the spectral efficiency can be significantly improved by adaptive RF spectral assignments because of the mitigation of both the channel fading effects and the backscattering effects. To rigorously verify such a statement, we compare the maximum achievable available aggregated signal transmission capacities of the hybrid IMDD OFDM-DFMA PONs with adaptive RF spectral assignment and fixed RF spectral assignments. Two Three different cases are considered for the fixed RF spectral assignments. In Case I, all available RF bandwidths are is-allocated for the upstream communications for measuring the impacts of the channel fading effects. For Case II, all available RF bandwidths are is assigned for inter-ONU communications for evaluating the impacts of the backscattering effects. For the above two three fixed RF spectral assignment cases, based on the experimental setup described in Section III.A, the signal transmission capacity of each channel and their resulting aggregated transmission capacities are plotted in Fig. 11. We can see that the adaptive RF spectral assignments (referred to as Case III) can achieve the highest aggregated signal transmission capacity. For Cease II, some subcarriers of channel 2 suffer the Brillouin backscattering effect, as shown which can be observed-in the RF spectrum in Fig. 4. Therefore, even with shorter transmission distances, the signal transmission capacity of €Case II is still relatively a little-lower than that of Case III. In comparison with the case of assigning all available bandwidths for the upstream (inter-ONU) communications, the adaptive RF spectral assignments can improve the aggregated signal transmission capacity by $\sim 31.4 \%(\sim 1.34 \%)$.

\section{CONCLUSIONS}

By making simple alterations to the remote node, a novel IMDD hybrid SSB OFDM-DFMA PON capable of supporting concurrent inter-ONU communications has been proposed and experimentally demonstrated with an aggregated net signal transmission capacity of $101.6 \mathrm{Gbit} / \mathrm{s}$ over $25 \mathrm{~km}$ SSMFs. The experimental results show that the IMDD hybrid SSB OFDM-DFMA PONs with concurrent inter-ONU communications have high tolerance to transmission system impairments and ONU channel interferences. Using the identified optimum digital filter length of 32 for both the upstream and inter-ONU communications, the power penalties caused by fiber transmissions and ONU channel interference are $\angle 1 \mathrm{~dB}$ and $2 \mathrm{~dB}$, respectively. With the hybrid SSB OFDM-DFMA PON techniques, flexible upstream and inter-ONU communication channel assignments can be conducted made-dynamically and adaptively according to the transmission system/network characteristics, traffic status and end-user requirements to achieve a high overall spectral efficiency. With adaptive RF spectral assignments, both the channel fading effects and the backscattering effects are mitigated, thus resulting in $>30 \%$ aggregated signal transmission capacity improvements. The low-cost effective passive remote node providing dedicated optical links for the inter-ONU communications; considerably reduces the signal propagation time by $90 \%$. The trade-off between ONU launch power dynamic range and aggregated signal transmission capacity has been investigated, which showsing that every $\sim 1 \mathrm{~dB}$ increase in ONU launch power dynamic range results in a $\sim 5 \mathrm{Gbit} / \mathrm{s}$ reduction in aggregated transmission capacity. In addition, for DSP-based signal transmission techniques, the speed of DACs and ADCs are important for achieving high signal transmission capacities. The proposed technique has the potential of allowing low-speed DACs/ADCs to be utilized to produce/receive targeted baseband signals, which can then be up-converted to the targeted sub-bands by low-cost electrical components. This could significantly reduce the overall ONU transceiver cost and is expected to offer a highly promising solution for flexible, low latency and high bandwidth access networks, required for $5 \mathrm{G}$ and beyond networks.

\section{REFERENCES}

[1] M. Huang, Y. Chen, P. Peng, H. Wang, and G. Chang, "A full field-of-view self-steering beamformer for $5 \mathrm{G} \mathrm{mm}$-wave fiber-wireless mobile fronthaul," J. Light. Technol., vol. 38, no. 6, pp. 1221-1229, 2020.

[2] A. O. Mufutau, F. P. Guiomar, M. A. Fernandes, A. Lorences-Riesgo, A. Oliveira, and P. P. Monteiro, "Demonstration of a hybrid optical fiberwireless 5G fronthaul coexisting with end-to-end 4G networks," J. Opt. Commun. Netw., vol. 12, no. 3, pp. 72-78, 2020.

[3] H. Zeng, X. Liu, S. Megeed, N. Chand, and F. Effenberger, "Real-time demonstration of CPRI-compatible efficient mobile fronthaul using FPGA," J. Light. Technol., vol. 35, no. 6, pp. 1241-1247, 2017.
Formatted: Underline, Font color: Red Formatted: Underline, Font color: Red

Formatted: Font color: Red 
[4] L. M. P. Larsen, A. Checko, and H. L. Christiansen, "A survey of the functional splits proposed for $5 \mathrm{G}$ mobile crosshaul networks," IEEE Commun. Surv. Tut. vol. 21, no. 1, pp. 146-172, 2019.

[5] 3GPP, "Study on new radio access technology: Radio access architecture and interfaces, V14.0.0 (2017-03)," Sophia Antipolis, France, Rep. TR 38.801 .

[6] "Transport network support of IMT-2020/5G," ITU-T, Geneva, Switzerland, Rep. GSTR-TN5G, 2018.

[7] eCPRI Specification V1.1. Interface Specification, Common Public Radio Interface, Jan. 2018

[8] D. Wake, A. Nkansah, and N. J. Gomes, "Radio over fiber link design fo next generation wireless systems," J. Light. Technol., vol. 28, no. 16, pp. 2456-2464, 2010.

[9] P. Torres-Ferrera, S. Straullu, S. Abrate, and R. Gaudino, "Upstream and downstream analysis of an optical fronthaul system based on DSP-Assisted channel aggregation," J. Opt. Commun. Netw., vol. 9, no. 12, pp 1191-1201, 2017.

[10]H. Li, Q. Yang, S. Fu, M. Luo, X. Li, Z. He, P. Jiang, Y. Liu, and S. Yu, "Digital code-division multiplexing channel aggregation for mobile fronthaul architecture with low complexity," IEEE Photon. $J$. vol. 10, no. 2, pp. 7902710, 2018

[11] M. Sung, S. Cho, H. S. Chung, S. M. Kim, and J. H. Lee, "Investigation of transmission performance in multi-IFoF based mobile fronthaul with dispersion-induced intermixing noise mitigation," Opt. Express, vol. 25, no. 8, pp. 9346-9357, 2017.

[12]M. Bolea, R. P. Giddings, M. Bouich, C. Aupetit-Berthelemot, and J. M. Tang, "Digital filter multiple access PONs with DSP-enabled software reconfigurability," J. Opt. Commun Netw, vol. 7, no 4, pp. 215-222, 2015.

[13]P. Assimakopoulos, S. Noor, M. Wang, H. Abdulsada, L. A. Neto, N. Genay, P. Chanclou, and N. J. Gomes, "Flexible and efficient DSP-assisted
subcarrier multiplexing for an analog mobile fronthaul," IEEE Photon. subcarrier multiplexing for an analog mobile
Technol. Lett, vol.35, no. 5, pp. 267-270, 2021

[14]X. Liu, H. Zeng, N. Chand, and F. Effenberger, "Efficient mobile fronthau via DSP-based channel aggregation," J. Light. Technol., vol. 34, no. 6, pp. $1556-1564,2016$

[15]E. Al-Rawachy, R. P. Giddings, and J. M. Tang, "Experimental demonstration of a DSP-based cross-channel interference cancellation technique for application in digital filter multiple access PONs," Opt. Express, vol. 25, no. 4, pp. 3850-3862, 2017

[16]J. Zou, S. A. Sasu, M. Lawin, A. Dochhan, J. Elbers, and M. Eiselt, "Advanced optical access technologies for next-generation $(5 \mathrm{G})$ mobile "Advanced optical access technologies for next-generation (5G) mobile
networks," J. Opt. Commun. Netw., vol. 12, no.10, pp. D86-D98, 2020.

[17]X. Pang, O. Ozolins, R. Lin, L. Zhang, A. Udalcovs, L. Xue, R. Schatz, U. Westergren, S. Xiao, W. Hu, G. Jacobsen, S. Popov, and J. Chen, "200 Gbps/Lane IM/DD technologies for short reach optical interconnects," $J$ Light. Technol., vol. 38, no. 2, pp. 492-503, 2020.

[18]W. Jin, A. Sankoh, Y. X. Dong, Z. Q. Zhong, R. P. Giddings, M O'Sullivan, J. Lee, T. Durrant, and J. M. Tang, "Hybrid SSB OFDM-digital filter multiple access PONs," J. Light. Technol., vol. 38, no. 8, pp.2095-2105, 2020.

[19]I. Parvez, A. Rahmati, I. Guvenc, A. I. Sarwat and H. Dai, "A survey on low latency towards 5G: RAN, core network and caching solutions," IEEE Commun. Surv. Tut. vol. 20, no. 4, pp. 3098-3130, 2018.

[20]X. Hu, L. Zhang, P. Cao, G. Zhou, F. Li and Y. Su, "Reconfigurable and scalable all-optical VPN in WDM PON," IEEE Photon. Technol. Lett., vol. 23, no. 14, pp. 941-943, 2011.

[21]R. Agrawal, A. Bedekar, T. Kolding, and V. Ram, "Cloud RAN challenge and solutions," Ann. Telecommun., vol. 72, no. 7, pp. 387-400, 2017.

[22]X. Hu, P. Cao, L. Zhang, X. Jiang, Z. Zhuang, and Y. Su, "Flexible and concurrent all-optical VPN in OFDMA PON," IEEE Photon. J., vol. 5 , no. 6, pp. 7902707, 2013 .

[23]C. Zhang, J. Huang, C. Chen, and K. Qiu, "All-optical virtual private network and ONUs communication in optical OFDM-based PON system," Opt. Express, vol. 19, no. 24, pp. 24816-24821, 2011.

[24]X. Li, C. Gan, Y. Yan, and H. Qiao, "Grid architecture of a metro-acces optical network to support discretionary peer-to-peer intracommunication and intercommunication between ONUs," J. Opt. Commun. Netw., vol.11, no. 3, pp. 130-139, 2019

[25]Y. Tian, T. Ye, and Y. Su, "Demonstration and scalability analysis of all-optical virtual private network in multiple passive optical network using ASK/FSK format," IEEE Photon. Technol. Lett., vol. 19, no. 20, pp. $1595-1597,2007$

[26]Z. Zhong, W. Jin, S. Jiang, J. He, D. Chang, R. Giddings, Y. Hong, M. O'Sullivan, T. Durrant, G. Mariani, J. Trewern, and J. Tang,
"Experimental demonstrations of concurrent adaptive inter-ONU and upstream communications in IMDD hybrid SSB OFDM-DFMA PONs," Opt. Fiber Commun. Conf., F4I.5, USA, 2021.

[27] L. Marple, "Computing the discrete-time "analytic" signal via FFT," IEEE Trans. Signal Process., vol. 47, no. 9, pp. 2600-2603, 1999.

[28]W. Jin, Z Q Zhong, J. X. He, A. Sankoh, R. P. Giddings, Y. H. Hong, I. Pierce, M. O'Sullivan, C. Laperle, J. Lee, G. Mariani, T. Durrant, and J. M. Tang, "Experimental demonstrations of hybrid OFDM-digital filter multiple access PONs," IEEE Photon. Technol. Lett., vol. 32, no. 13, pp. 751-754, 2020.

29]M. L. Deng, A. Sankoh, R. P. Giddings, and J. M. Tang, "Experimental demonstrations of $30 \mathrm{~Gb} / \mathrm{s} / \lambda$ digital orthogonal filtering-multiplexed multiple channel transmissions over IMDD PON systems utilizing 10G-class optical devices," Opt. Express, vol. 25, no. 20, pp. 24251-24261, 2017.

30]Z. Q. Zhong, W. Jin, Y. X. Dong, A. Sankoh, J. X. He, Y. H. Hong, R. P. Giddings, I. Pierce, M. O'sullivan, J. Lee, G. Mariani, T. Durrant, and J. M. Tang, "Experimental demonstrations of matching filter-free digital filter multiplexed SSB OFDM IMDD transmission systems," IEEE Photon. J., vol. 13, no. 2, pp. 7900512, 2021. 